\title{
Real multiplication through explicit correspondences
}

\author{
Abhinav Kumar and Ronen E. Mukamel
}

\begin{abstract}
We compute equations for real multiplication on the divisor classes of genus- 2 curves via algebraic correspondences. We do so by implementing van Wamelen's method for computing equations for endomorphisms of Jacobians on examples drawn from the algebraic models for Hilbert modular surfaces computed by Elkies and Kumar. We also compute a correspondence over the universal family for the Hilbert modular surface of discriminant 5 and use our equations to prove a conjecture of A. Wright on dynamics over the moduli space of Riemann surfaces.
\end{abstract}

\section{Introduction}

Abelian varieties, their endomorphisms and their moduli spaces play a central role in modern algebraic geometry and number theory. Their study has important applications in a broad array of fields, including cryptography, dynamics, geometry, and mathematical physics. Of particular importance are the abelian varieties with extra endomorphisms (other than those in $\mathbb{Z}$ ). In dimension 1 , elliptic curves with complex multiplication have been studied extensively. In this paper, we focus on curves of genus 2 whose Jacobians have real multiplication by a real quadratic ring $\mathcal{O}$.

For such a curve $C$ over a number field $K$, we use the ideas from van Wamelen's work [18-20] to explicitly compute the action of real multiplication by $\mathcal{O}$ on the divisors of $C$. In particular, we determine equations for an algebraic correspondence on $C$, that is, a curve $Z$ with two maps $\phi, \psi: Z \rightarrow C$ such that the induced endomorphism $T=\psi_{*} \circ \phi^{*}$ of $\operatorname{Jac}(C)$ generates $\mathcal{O}$. The discovery of the correspondence $Z$ uses floating-point calculations on the analytic Jacobian $\operatorname{Jac}(C) \otimes \mathbb{C}$. We then rigorously certify the real multiplication of $\mathcal{O}$ on $\operatorname{Jac}(C)$ by computing the action of $T$ on 1-forms using exact arithmetic in $K$, or a small degree extension of $K$. Combined with standard equations for the group law on $\operatorname{Jac}(C)$, our techniques immediately lead to an algebraic description the action of $\mathcal{O}$ on degree- 0 divisors of $C$.

This paper completes a research program initiated in $[4,11]$. Let $\mathcal{M}_{g, n}$ denote the moduli space of smooth genus- $g$ curves with $n$ marked points, and, for each totally real order $\mathcal{O}$, let $\mathcal{M}_{g, n}(\mathcal{O})$ denote the locus of curves whose Jacobians admit real multiplication by $\mathcal{O}$. The paper [4] describes a method for parametrizing the Humbert surface $\mathcal{M}_{2}(\mathcal{O})=\mathcal{M}_{2,0}(\mathcal{O})$ for real quadratic $\mathcal{O}$ as well as its double cover, the Hilbert modular surface $Y(\mathcal{O})$. It also carries out the computation for $\mathcal{O}=\mathcal{O}_{K}$, the ring of integers of every real quadratic field $K$ of discriminant less than 100, producing equations for the corresponding Hilbert modular surfaces. The paper [11] describes a method for computing the action of $\mathcal{O}$ on the 1 -forms of curves in $\mathcal{M}_{2}(\mathcal{O})$, and uses it in particular to compute algebraic models for Teichmüller curves $\mathcal{M}_{2}$. Using these techniques, one can furnish equations defining curves $C \in \mathcal{M}_{2}(\mathcal{O})$, rigorously prove that $\operatorname{Jac}(C)$ admits real multiplication by $\mathcal{O}$, and rigorously compute the action of $\mathcal{O}$ on

Received 10 February 2016.

2010 Mathematics Subject Classification 14-04, 14H40 (primary), 32G15, $14 \mathrm{G} 35$ (secondary).

Contributed to the Twelfth Algorithmic Number Theory Symposium (ANTS-XII), Kaiserslautern, Germany, 29 August-2 September 2016. 
the 1-forms of $C$. In this paper we solve the problem of describing the action of $\mathcal{O}$ on $\operatorname{Jac}(C)$ as algebraic morphisms by computing the action on the divisors of $C$.

Example for discriminant 5. To demonstrate our method, consider the genus-2 curve

$$
C: u^{2}=t^{5}-t^{4}+t^{3}+t^{2}-2 t+1 \text {. }
$$

Equation (1.1) was obtained from the equations in [4]. Using the methods of this paper, we can formulate and prove the following theorem.

Theorem 1. Let $\phi: Z \rightarrow C$ be the degree-2 cover of $C$ in equation (1.1) defined by

$$
t^{2} x^{2}-x-t+1=0 .
$$

The curve $Z$ is of genus 6 and admits an additional map $\psi: Z \rightarrow C$ of degree 2. The induced endomorphism $T=\psi_{*} \circ \phi^{*}$ of $\operatorname{Jac}(C)$ is self-adjoint with respect to the Rosati involution, satisfies $T^{2}-T-1=0$, and generates real multiplication by $\mathcal{O}_{5}$.

In $\S 5$ we give several other examples of correspondences on particular genus- 2 curves of varying complexity. In $\S 6$, we describe how to implement our method in families and compute a correspondence for the entire Hilbert modular surface $Y\left(\mathcal{O}_{5}\right)$.

Divisor classes supported at eigenform zeros. In the universal ${ }^{\dagger}$ Jacobian over the space $\mathcal{M}_{g, n}(\mathcal{O})$, there is a natural class of multisection obtained from $\mathcal{O}$-linear combinations of divisor classes supported at eigenform zeros and marked points. Filip recently showed that such divisors play a pivotal role in the behavior of geodesics in moduli space [6]. As an application of our equations for real multiplication, we prove a theorem about such divisors over $\mathcal{M}_{2,1}\left(\mathcal{O}_{5}\right)$ and prove a conjecture of Wright on dynamics over the moduli space of curves.

In the universal Jacobian over $\mathcal{M}_{2,1}\left(\mathcal{O}_{5}\right)$, let $L$ be the multisection whose values at the curve $C$ marked at $P \in C$ are divisors of the form

$$
\left(P-Z_{1}\right)-T \cdot\left(Z_{2}-Z_{1}\right) \in \operatorname{Jac}(C)
$$

where $T$ is a Rosati invariant endomorphism of $\operatorname{Jac}(C)$ satisfying $T^{2}-T-1=0$ and $Z_{1}$ and $Z_{2}$ are the zeros of a $T$-eigenform $\omega$ on $C$. The various choices of endomorphism $T$, eigenform $\omega$, and ordering of the zeros of $\omega$ give four points in $\operatorname{Jac}(C)$ for generic $(C, P) \in \mathcal{M}_{2,1}\left(\mathcal{O}_{5}\right)$. The vanishing of $L$ defines a closed subvariety of $\mathcal{M}_{2,1}\left(\mathcal{O}_{5}\right)$ :

$$
\mathcal{M}_{2,1}\left(\mathcal{O}_{5} ; L\right)=\left\{(C, P) \in \mathcal{M}_{2,1}\left(\mathcal{O}_{5}\right): \begin{array}{l}
\text { some branch of } L \\
\text { vanishes at }(C, P)
\end{array}\right\}
$$

One might expect $\mathcal{M}_{2,1}\left(\mathcal{O}_{5} ; L\right)$ to be a curve in the 3 -fold $\mathcal{M}_{2,1}\left(\mathcal{O}_{5}\right)$ since the relative dimension of the universal Jacobian is 2 . We use our equations for real multiplication to show that $\mathcal{M}_{2,1}\left(\mathcal{O}_{5} ; L\right)$ is unexpectedly large.

Theorem 2. The space $\mathcal{M}_{2,1}\left(\mathcal{O}_{5} ; L\right)$ is an irreducible surface in $\mathcal{M}_{2,1}$.

To relate Theorem 2 to dynamics, recall that $\mathcal{M}_{g, n}$ carries a Teichmüller metric and that every vector tangent to $\mathcal{M}_{g, n}$ generates a complex geodesic, that is, a holomorphic immersion $\mathbb{H} \rightarrow \mathcal{M}_{g, n}$ which is a local isometry. McMullen proved that the locus $\mathcal{M}_{2}(\mathcal{O})$ is the closure of

\footnotetext{
†Technically, it would be more accurate to use the term 'universal' to describe the family over the stack whose underlying coarse moduli space is the Hilbert modular surface. However, we will indulge in this mild abuse of terminology throughout this paper.
} 
a complex geodesic in the moduli space $\mathcal{M}_{2}$ of unmarked genus-2 curves for each real quadratic $\mathcal{O}[\mathbf{1 2}]$. A corollary of Theorem 2 shows that $\mathcal{M}_{2,1}\left(\mathcal{O}_{5} ; L\right)$ enjoys the same property.

TheOrem 3. There is a complex geodesic $f: \mathbb{H} \rightarrow \mathcal{M}_{2,1}$ with $\overline{f(\mathbb{H})}=\mathcal{M}_{2,1}\left(\mathcal{O}_{5} ; L\right)$.

In other words, there is a dynamically natural way to choose finitely many points on each curve in $\mathcal{M}_{2}\left(\mathcal{O}_{5}\right)$. This was originally conjectured by Wright, and will be proven by other means in the forthcoming paper [5].

By Filip's characterization of the behavior of complex geodesics in moduli space [6], every complex geodesic is dense in a subvariety of $\mathcal{M}_{g, n}(\mathcal{O})$ characterized by $\mathcal{O}$-linear relations among divisor classes supported at eigenform zeros and marked points. Theorem 3 is the first example of such a subvariety where a relation involving a ring strictly larger than $\mathbb{Z}$ appears.

Prior work on equations for real multiplication. Several authors $[\mathbf{7}, \mathbf{8}, \mathbf{1 4}, \mathbf{1 5}, \mathbf{2 1}, \mathbf{2 2}]$ have given geometric descriptions of real multiplication based on Humbert's work on Poncelet configurations of conics $[\mathbf{9}, \mathbf{1 0}, \mathbf{1 7}]$. Our work combines explicit examples or families of genus-2 curves (obtained from the equations for Hilbert modular surfaces computed in [4]) with the method of van Wamelen outlined in $[\mathbf{1 9}, \S 3]$. The latter uses high-precision numerical sampling on the Jacobian and subsequent linear algebra to find explicit algebraic descriptions of isogenies for Jacobians of genus-2 curves. We describe the isogenies through correspondences; see [16] for other examples of equations for real multiplication obtained through correspondences. Furthermore, we address the challenge of computing correspondences in families, thereby giving a description of real multiplication for the universal abelian surface over a Hilbert modular surface.

Outline. In $\S 2$ we recall some basic facts about Jacobians of curves, their endomorphisms and correspondences. In $\S 3$ we describe our method for finding the equations of a correspondence associated to a Jacobian endomorphism. In $\S 4$ we describe how to compute the induced action on 1-forms and thereby certify the equations obtained by the method in $\S 3$. In $\S 5$ we give several examples of varying complexity of explicit correspondences. In $\S 6$ we address challenges to implementing our algorithm in families, and describe a correspondence for the entire Hilbert modular surface for discriminant 5 . In $\S 7$ we discuss the applications to dynamics and prove Theorems 2 and 3.

Computer files. Auxiliary files containing computer code to verify the calculations in this paper are available from http://arxiv.org/abs/1602.01924. To access these, download the source file for the paper. It is a tar archive, which can be extracted to produce not only the $\mathrm{AT}_{\mathrm{E} X}$ file for this paper, but also the computer code. The text file README.txt gives a brief guide to the various auxiliary files.

\section{Background}

In this section we recall some general facts about curves, their Jacobians and algebraic correspondences. We will work over the complex numbers. The basic reference for this section is $[1]$.

Jacobians. Let $C$ be a smooth projective curve of genus $g$ over $\mathbb{C}$. The holomorphic 1forms on $C$ form a $g$-dimensional vector space $\Omega(X)$. Integration gives rise to an embedding $H_{1}(C, \mathbb{Z}) \rightarrow \Omega(C)^{*}$ and the image of this embedding is a lattice. The quotient

$$
\operatorname{Jac}(C)=\Omega(C)^{*} / H_{1}(C, \mathbb{Z})
$$


is a compact, complex torus called the Jacobian of $C$. The symplectic intersection form on $H_{1}(C, \mathbb{Z})$ induces a principal polarization on the torus $\operatorname{Jac}(C)$ (that is, an isomorphism of this abelian variety with its dual).

The Abel-Jacobi map. $\quad$ Let $\operatorname{Pic}^{0}(C)$ denote the group of degree-0 divisors on $C$ up to linear equivalence. Integration gives rise to an isomorphism of groups

$$
A J: \operatorname{Pic}^{0}(C) \rightarrow \operatorname{Jac}(C) .
$$

This is the Abel-Jacobi map. When $\operatorname{Pic}^{0}(C)$ is thought of as the complex points of the Picard variety of $C$, this map is an isomorphism of abelian varieties over $\mathbb{C}$.

The theta divisor. Choosing a base point $P_{0} \in C$ allows us to define a birational map $\xi$ from the $g$ th symmetric power of $C$ to $\operatorname{Pic}^{0}(C)$ via the formula

$$
\xi\left(\left\{P_{1}, \ldots, P_{g}\right\}\right)=\left(\sum_{i} P_{i}\right)-g P_{0} .
$$

The divisor $\left\{S \in \operatorname{Sym}^{g}(C): P_{0} \in S\right\}$ gives rise to a divisor $\Theta$ on $\operatorname{Jac}(C)$ called the theta divisor.

Pullback and pushforward. Now consider a holomorphic map $\psi: Z \rightarrow C$ between curves. The map $\psi$ induces a map $\Omega(C) \rightarrow \Omega(Z)$ whose dual covers a holomorphic homomorphism

$$
\psi_{*}: \operatorname{Jac}(Z) \rightarrow \operatorname{Jac}(C) .
$$

Under the identification of Jacobians with degree-0 divisors via the Abel-Jacobi map, $\psi_{*}$ corresponds to the pushforward of divisors, that is,

$$
\psi_{*}\left(\sum_{i} P_{i}-\sum_{i} Q_{i}\right)=\sum_{i} \psi\left(P_{i}\right)-\sum_{i} \psi\left(Q_{i}\right) .
$$

We call $\psi_{*}$ the pushforward map. The map $\psi$ also induces a pullback map

$$
\psi^{*}: \operatorname{Jac}(C) \rightarrow \operatorname{Jac}(Z)
$$

obtained as the dual map to $\psi_{*}$ by identifying the Jacobians of $C$ and $Z$ with their corresponding duals via their principal polarizations. For non-constant $\psi$, we can obtain $\psi^{*}$ at the level of divisors by summing along fibers, that is,

$$
\psi^{*}\left(\sum_{i} P_{i}-\sum_{i} Q_{i}\right)=\sum_{i} \psi^{-1}\left(P_{i}\right)-\sum_{i} \psi^{-1}\left(Q_{i}\right)
$$

while for a constant map $\psi^{*}=0$. The composition $\psi_{*} \circ \psi^{*}$ is the multiplication by $\operatorname{deg}(\psi)$ map on $\operatorname{Jac}(C)$.

Correspondences. A correspondence $Z$ on $C$ is a holomorphic curve in $C \times C$. Fix a correspondence $Z$ on $C$ and let $\phi=\pi_{1}$ and $\psi=\pi_{2}$ be the the two projection maps from $Z$ to $C$. The correspondence $Z$ gives rise to an endomorphism of $\operatorname{Jac}(C)$ via the formula $T=\psi_{*} \circ \phi^{*}$. From equations (2.1) and (2.2), we see that $T$ acts on divisors of the form $P-Q$ by the formula

$$
T(P-Q)=\psi\left(\phi^{-1}(P)\right)-\psi\left(\phi^{-1}(Q)\right) .
$$

Such divisors generate $\mathrm{Pic}^{0}(C)$, so equation (2.3) determines the action of $T$ on $\mathrm{Pic}^{0}(C)$. 
Conversely, every endomorphism $T$ of the Jacobian endomorphism arises via a correspondence. To see this, we embed $C$ in $\operatorname{Jac}(C)$ via the map $P \mapsto P-P_{0}$ (note that when the genus of $C$ is 2 , the resulting cycle is just the theta divisor $\Theta$ ). Since the image of $C$ under this embedding generates the group $\operatorname{Jac}(C)$, the restriction of $T$ to $C$ determines $T$. This map $\left.T\right|_{C}$ is a $C$-valued point of $\operatorname{Jac}(C) \cong \operatorname{Pic}_{C}^{0}$, and by the functorial property of the Picard variety, it corresponds to a line bundle $\mathcal{L}$ on $C \times C$, whose fibers $\left.\mathcal{L}\right|_{C \times P}$ are all of degree 0 . Then we can take $Z$ to be an effective divisor corresponding to the line bundle $\mathcal{L} \otimes \pi_{1}^{*}\left(\mathcal{O}_{C}\left(g P_{0}\right)\right)$. Concretely, the intersection of $Z$ with $P \times C$ consists of points $\left(P, Q_{i}\right)$ with $Q_{1}, \ldots, Q_{g} \in C$ satisfying $T \cdot\left(P-P_{0}\right)=\sum_{i} Q_{i}-g P_{0}$. Using this fact, it is easily checked that the two constructions are inverse to each other.

\section{Computing equations for correspondences}

In this section we describe our method for discovering correspondences. The methods in this section are numerical and rely on floating point approximation. Nonetheless, the correspondences we obtain are presented by equations with exact coefficients lying in a number field. In $\S 4$ we will describe how to certify these equations using only rigorous integer arithmetic in number fields to prove theorems about real multiplication on genus-2 Jacobians.

Setup. Our starting point is a fixed curve $C$ of genus 2 known to have a Jacobian endomorphism $T$ generating real multiplication by the real quadratic order $\mathcal{O}_{D}$ of discriminant $D$. Such curves can be supplied by the methods in [4]. We assume that $C$ and $T$ are defined over a number field $K$ and that $C$ is presented as a hyperelliptic curve

$$
C: u^{2}=h(t) \quad \text { with } h \in K[t] \text { monic, } \operatorname{deg}(h)=5 \text {. }
$$

We fix an embedding $K \subset \mathbb{C}$ so that we can change base to $\mathbb{C}$ and work with the analytic curve $C^{\text {an }}$ and the analytic Jacobian $J^{\text {an }}=\operatorname{Jac}\left(C^{\text {an }}\right)$. For simplicity we have assumed in this section that $h$ is monic of degree 5 so that $C$ has a $K$-rational Weierstrass point $P_{0}$ at infinity. We discuss below how to handle the sextic case (see Remark 13).

Analytic Jacobians in Magma. The computer system Magma has several useful functions for working with analytic Jacobians and their endomorphisms, implemented by van Wamelen. An excellent introduction may be found in [19], and extensive documentation is available in the Magma handbook [2]. The relevant functions for us are as follows.

(a) AnalyticJacobian (see also BigPeriodMatrix): computes the periods of $d t / u$ and $t d t / u$ in $\Omega(C)$, yielding a numerical approximation to the period matrix $\Pi\left(C^{\text {an }}\right)$ and a model for the analytic Jacobian $J^{\text {an }}=\mathbb{C}^{2} / \Pi\left(C^{\text {an }}\right) \cdot \mathbb{Z}^{4}$.

(b) EndomorphismRing: computes generators for the endomorphism ring of $J^{\text {an }}$. Each endomorphism $T^{\text {an }}$ is presented as a pair of matrices $T_{\Omega}^{\mathrm{an}} \in M_{2}(\mathbb{C})$ and $T_{\mathbb{Z}}^{\mathrm{an}} \in M_{4}(\mathbb{Z})$ satisfying $\Pi\left(C^{\text {an }}\right) \cdot T_{\mathbb{Z}}=T_{\Omega} \cdot \Pi\left(C^{\text {an }}\right)$ (up to floating point precision).

(c) ToAnalytic Jacobian: computes the Abel-Jacobi map by numerical integration.

(d) FromAnalyticJacobian: computes the inverse of the Abel-Jacobi map using theta functions.

Discovering correspondences. We compute equations defining the correspondence $Z$ on $C$ associated to $T$ as follows.

(1) Compute the analytic Jacobian $J^{\text {an }}$ and an endomorphism $T^{\text {an }}$ generating real multiplication.

(2) Choose low height points $P_{i}=\left(t_{i}, u_{i}\right) \in C^{\text {an }}$ with $t_{i} \in K$. 
(3) For each $i$, numerically compute points $R_{i}=\left(t\left(R_{i}\right), u\left(R_{i}\right)\right)$ and $Q_{i}=\left(t\left(Q_{i}\right), u\left(Q_{i}\right)\right)$ in $C^{\text {an }}$ such that

$$
A J\left(Q_{i}+R_{i}-2 P_{0}\right)=T^{\mathrm{an}} \cdot\left(A J\left(P-P_{0}\right)\right) .
$$

(4) For each $i$, compute the exact coefficients of the polynomial $F_{i}(x)=\left(x-t\left(Q_{i}\right)\right)\left(x-t\left(R_{i}\right)\right)$ in $K[x]$ using LLL.

(5) Interpolate to determine a polynomial $F \in K(t)[x]$ which specializes to $F_{i}$ at $t=t\left(P_{i}\right)$ and let $Z$ be the degree- 2 cover defined by $F$, that is, with

$$
K(Z)=K(C)[x] /(F) .
$$

To realize $Z$ as a divisor in $C \times C$, we need to compute a square root for $h(x)$ in $K(Z)$. For small examples, this can be done by working in the function field of $Z$. In general, we revisit steps (3) and (4) and do the following.

(6) For each $i$, determine $u\left(Q_{i}\right)$ as a $K$-linear combination of $u\left(P_{i}\right)$ and $u\left(P_{i}\right) t\left(Q_{i}\right)$.

(7) Interpolate to determine a rational function $y \in K(Z)$ which is a $K(t)$-linear combination of $u$ and $u x$ and equals $u\left(Q_{i}\right)$ when specialized to $(t, u, x)=\left(t\left(P_{i}\right), u\left(P_{i}\right), t\left(Q_{i}\right)\right)$.

REMARK 4. Typically we use AnalyticJacobian and EndomorphismRing to carry out step (1), and ToAnalyticJacobian and FromAnalyticJacobian to carry out step (3). The remainder of the algorithm requires only the matrix $T_{\Omega}^{\text {an }}$ (and not $T_{\mathbb{Z}}^{\text {an }}$ ) which could also be obtained using the algorithm in [11] rather than EndomorphismRing.

REMARK 5. We do not carry out a detailed analysis of the floating point precision needed or the running time of our algorithm. We remark that 400 digits of precision were sufficient for the examples in this paper and that the machine used to perform the computations in this paper ( $4 \mathrm{GHz}, 32 \mathrm{~GB} \mathrm{RAM})$ completed the entire sampling and interpolation process for individual correspondences in minutes. For our most complicated example, presented in Theorem 12, CPU time was under 2 minutes.

To be able to carry out these steps, we need a large supply of sample points, and sufficient precision. As far as $Z$ is concerned the number of sample points needed for interpolation to find the equation of $Z$, we closely follow the argument of $[\mathbf{1 8}, \S 3]$. There it is observed that the coefficients of $F$ (which are $x_{1}+x_{2}$ and $x_{1} x_{2}$ in the notation of [18]) are rational functions in $t$ and have degrees which are bounded by the intersection number of $\alpha(\Theta)$ and $2 \Theta$. In our case, this equals $\operatorname{tr}_{\mathbb{Q}(\sqrt{D}) / \mathbb{Q}}\left(\alpha^{2}\right)$. Consequently, we choose $\alpha \in \mathcal{O}_{D}$ for which the trace is minimized: $\alpha= \pm \sqrt{D} / 2$ if $D$ is even, and $\alpha=( \pm 1 \pm \sqrt{D}) / 2$ if $D$ is odd. In practice, since the degrees of the functions involved may be quite a bit smaller than the upper bound, it is more efficient in terms of both time and computer memory to choose a small sample size and attempt to see if the computation of $Z$ succeeds.

REMARK 6. From the equations for $Z$ and the maps $\phi, \psi$ to $C$, we can compute the action of $T$ on divisors of the form $P-Q$ using equation (2.3). We can then use standard equations [3] for the group law on $\operatorname{Jac}(C)$ to extend this formula to arbitrary divisor classes of degree 0 . Similarly, we can compute the algebraic action of an arbitrary element $m+n T \in \mathbb{Z}[T]$ of the real quadratic order using formulas for the group law.

Example. We conclude this section with an example for discriminant 5 . Let $K=\mathbb{Q}$ and let $C$ be the genus- 2 curve defined by

$$
C: u^{2}=h(t) \quad \text { where } h(t)=t^{5}-t^{4}+t^{3}+t^{2}-2 t+1 .
$$

The Jacobian of $C$ corresponds to the point $(g, h)=\left(-\frac{8}{3}, \frac{47}{2}\right)$ in the model computed in [4] for the Hilbert modular surface $Y\left(\mathcal{O}_{5}\right)$ parametrizing principally polarized abelian surfaces with 
an action of $\mathcal{O}_{5}$. By the method outlined above, we discover that the degree-2 branched cover $f: Z \rightarrow C$ defined by

$$
F(x)=0 \quad \text { where } F(x)=t^{2} x^{2}-x-t+1 \in K(C)[x]
$$

is a correspondence associated to real multiplication by $\mathcal{O}_{5}$. In fact, setting

$$
y=\frac{1}{t^{3}} u-\frac{t+1}{t^{3}} u x \in K(Z)
$$

we find that $y$ is a square root of $h(x)$ in $K(Z)$, and the map $\psi(t, u, x)=(x, y)$ defines a second map $Z \rightarrow C$. In $\S 4$ we will prove that $T=\psi_{*} \circ \phi^{*}$ generates real multiplication by $\mathcal{O}_{5}$, thereby certifying these equations for $Z$. We note here that the degree of $\psi$ is 2 since, fixing $x$, there are two choices for $t$ satisfying equation (3.4), and $u$ is determined by $(x, y, t)$ by equation (3.5). The curve $Z$ has genus 6 , as can be readily computed in Magma or Maple.

REMARK 7. It would be interesting to use the tools in this paper to study the geometry of correspondences over Hilbert modular surfaces. In particular, one might explore how the geometry of $Z$ varies with $C$ and $T$, and how $Z$ specializes at curves $C$ lying on arithmetically and dynamically interesting loci such as Teichmüller curves and Shimura curves. For instance, compare Theorems 1 and 14 for discriminant 5 and Theorems 8 and 10 for discriminant 12 .

\section{Minimal polynomials and action on 1-forms}

In this section we describe how to certify the equations we discovered by the method in $\S 3$. We have now determined an equation for a curve $Z$ with an obvious degree-2 map $\phi: Z \rightarrow C$ given by $\phi(t, u, x)=(t, u)$. We have also computed equations for a second map $\psi: Z \rightarrow C$ given by $\psi(t, u, x)=(x, y)$.

We will now describe how to compute the action $T_{\Omega}$ of $T=\psi_{*} \circ \phi^{*}$ on $\Omega(C)$. Since the representation of the endomorphism ring of $\operatorname{Jac}(C)$ on $\Omega(C)$ is faithful, the minimal polynomial for $T$ is equal to the minimal polynomial for $T_{\Omega}$. Fixing $\omega \in \Omega(C)$, to compute $T_{\Omega}(\omega)$, we first pull back along $\psi$ and then push forward along $\phi$. The order of composition is reversed since the functor sending $C$ to the vector space $\Omega(C)$ is contravariant, whereas the functor sending $C$ to $\operatorname{Jac}(C)$ is covariant. Pullbacks are straightforward, and the pushforward along $\phi$ can be computed from the rule

$$
\phi_{*}(v \eta)=\operatorname{tr}(v) \omega \quad \text { when } \eta=\phi^{*}(\omega) \text { and } v \in K(Z) .
$$

The trace on the right-hand side is with respect to the field extension $K(Z)$ over the subfield isomorphic to $K(C)$ associated to the map $\phi$. We now see that

$$
T_{\Omega}(\omega)=\phi_{*} \circ \psi^{*}(\omega)=\operatorname{tr}\left(\psi^{*}(\omega) / \phi^{*}(\omega)\right) \cdot \omega .
$$

The trace on the right-hand side of equation (4.2) is over the field extension $K(t, u, x) / K(t, u)$ and can be computed easily from the equations defining $\phi$. We return to the example in the previous section.

ExAmple. Let $Z \subset C \times C$ be the correspondence defined by equations (3.3)-(3.5). Let $\omega_{1}=d t / u$ and $\omega_{2}=t d t / u$ be the standard basis for $\Omega(C)$. To compute the action of $T_{\Omega}$ on $\Omega(C)$, we need to work with the function field $K(Z)$ and its derivations. The derivations form a one-dimensional vector space over $K(Z)$. It is spanned by both $d x$ and $d t$, and the relation between them is computed by implicitly differentiating equation (3.4). We compute

$$
\frac{\psi^{*}\left(\omega_{1}\right)}{\phi^{*}\left(\omega_{1}\right)}=\frac{d x / y}{d t / u}=\frac{\left(-2 t^{4}+t^{3}-t^{2}\right) x+\left(-2 t^{4}+4 t^{3}-t^{2}-t+1\right)}{4 t^{3}-4 t^{2}+1} .
$$


We now need to compute the trace of the right-hand side over $K(t, u)$. From equation (3.4), we see that the trace of $x$ is $1 / t^{2}$, and therefore the trace of the right-hand side of equation (4.3) is $1-t$. We conclude that

$$
T_{\Omega}(d t / u)=(1-t) d t / u .
$$

Similarly, we compute that $T_{\Omega}(t d t / u)=-d t / u$ and hence the matrix for $T_{\Omega}$ is

$$
M=\left(\begin{array}{cc}
1 & -1 \\
-1 & 0
\end{array}\right)
$$

The minimal polynomial for $M$, hence for $T_{\Omega}$ and $T$ as well, is $T^{2}-T-1$. We conclude that $T$ generates a ring $\mathbb{Z}[T]$ isomorphic to $\mathcal{O}_{5}$.

Rosati involution. The adjoint for $T$ with respect to the Rosati involution is the endomorphism

$$
T^{\dagger}=\left(\psi_{*} \circ \phi^{*}\right)^{\dagger}=\phi_{*} \circ \psi^{*} .
$$

By computing the action of $T^{\dagger}$ on $\Omega(C)$ by the procedure above, we verify that $T_{\Omega}^{\dagger}=T_{\Omega}$ and conclude that $T=T^{\dagger}$ is self-adjoint with respect to the Rosati involution.

We note that the proof of Theorem 1 stated in the introduction is now complete.

\section{Further examples}

We now describe several other examples of the results one can obtain via our method. We choose relatively simple curves and small discriminants for purposes of illustration. For instance, the first two examples have Weierstrass points at $\infty$, and the others have two rational points at $\infty$. Each of the theorems stated in this section is proved by carrying out an analysis similar to our analysis of the curve defined by equation (3.3) in $\S 3$ and $\S 4$. We provide computer code in the auxiliary files to carry out these analyses.

Our first example involves a quadratic ring of slightly larger discriminant.

Theorem 8. Let $C$ be the curve defined by $u^{2}=t^{5}-6 t^{4}+15 t^{3}-22 t^{2}+17 t$ and let $\phi: Z \rightarrow C$ be the degree-2 branched cover defined by

$t\left(t^{2}-3 t+1\right)^{2} x^{2}-\left(4 t^{5}-23 t^{4}+46 t^{3}-37 t^{2}+6 t+17\right) x+4 t\left(t^{4}-6 t^{3}+15 t^{2}-22 t+17\right)=0$.

The curve $Z$ is of genus 12 and admits a map $\psi: Z \rightarrow C$ of degree 5 . The induced endomorphism $T=\psi_{*} \circ \phi^{*}$ of $\operatorname{Jac}(C)$ satisfies $T^{2}-3=0$ and generates real multiplication by $\mathcal{O}_{12}$.

Remark 9. The curve $C$ in Theorem 8 corresponds to the point $(e, f)=\left(\frac{34}{27},-\frac{5}{3}\right)$ on $Y\left(\mathcal{O}_{12}\right)$ in the coordinates of [4]. The proof of the theorem proceeds along similar lines to that of Theorem 1 . The map $\psi: Z \rightarrow C$ takes $(t, u, x)$ to $(x, y)$ where

$$
y=-\frac{t^{6}-5 t^{5}+12 t^{4}-21 t^{3}+32 t^{2}-17 t-17}{t^{2}\left(t^{2}-3 t+1\right)^{3}} x u+\frac{2(t-2)\left(t^{4}-2 t^{3}-t^{2}-2 t+17\right)}{t\left(t^{2}-3 t+1\right)^{3}} u .
$$

The function field of $Z$ is generated by $x$ and $u$. We depict a plane model for $Z$ in Figure 1 .

Our next example illustrates how the method developed in this paper may be used to identify eigenforms and determine points on Teichmüller curves. Recall that $W_{D}$ is the moduli space of genus-2 eigenforms for $\mathcal{O}_{D}$ with a double zero, and is a disjoint union of Teichmüller curves [12]. 


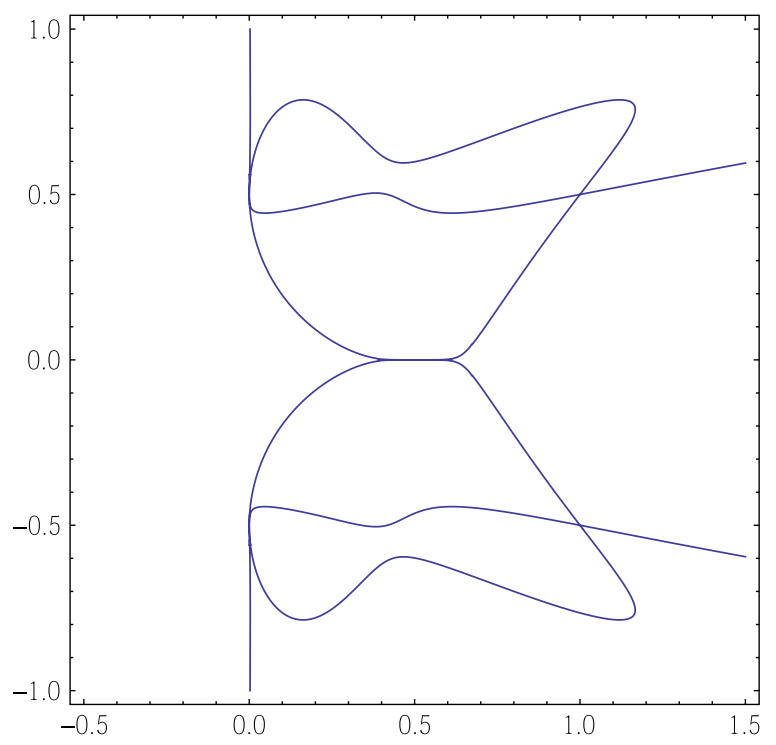

Figure 1. We plot the curve $Z$ of Theorem 8 in the $(1 / x, 1 / u)$-plane.

Theorem 10. Let $C$ be the curve $u^{2}=t^{5}-2 t^{4}-12 t^{3}-8 t^{2}+52 t+24$ and let $\phi: Z \rightarrow C$ be the degree-2 branched cover defined by

$$
\begin{aligned}
& 16(t-2)(t+1)^{2} x^{2}-\left(3 t^{4}+16 t^{3}+12 t^{2}-192 t-164\right) x \\
& \quad+\left(9 t^{5}-12 t^{4}-140 t^{3}-48 t^{2}+276 t+16\right)=0 .
\end{aligned}
$$

The curve $Z$ is of genus 11 and admits a map $\psi: Z \rightarrow C$ of degree 5 . The induced endomorphism $T=\psi_{*} \circ \phi^{*}$ of $\operatorname{Jac}(C)$ satisfies $T^{2}-3=0$ and generates real multiplication by $\mathcal{O}_{12}$. Moreover, the moduli point corresponding to $C$ on $Y\left(\mathcal{O}_{12}\right)$ lies on the WeierstrassTeichmüller curve $W_{12}$.

Remark 11. The curve $C$ in Theorem 10 was shown in [4] to have a Jacobian which admits real multiplication by $\mathcal{O}_{12}$. In fact, it corresponds to the point $(e, f)=\left(-\frac{3}{8},-\frac{1}{2}\right)$ on $Y\left(\mathcal{O}_{12}\right)$ in the coordinates of [4]. In [11], we showed using the eigenform location algorithm that $d t / u$ is an eigenform for $\mathcal{O}_{12}$ to conclude that this point lies on $W_{12}$. The equations for $Z$ and the maps $\phi, \psi$ yield an alternate proof of both of these facts. For completeness, we note that the expression for the rational function $y$ on $Z$ needed to define $\psi:(t, u, x) \mapsto(x, y)$ is

$$
y=-\frac{11 t^{4}-24 t^{3}+12 t^{2}-112 t-132}{64(t-2)^{2}(t+1)^{3}} x u \sqrt{3}-\frac{15 t^{5}-28 t^{4}-36 t^{3}+288 t^{2}-52 t-144}{64(t-2)^{2}(t+1)^{3}} u \sqrt{3}
$$

The rest of the verification is carried out in the computer code.

Our next example involves a genus-2 curve without a rational Weierstrass point. The resulting correspondence is more complicated, but still well within the reach of our method.

Theorem 12. Let $C$ be the curve $u^{2}=t^{6}+t^{5}+7 t^{2}-5 t+4$, and let $\phi: Z \rightarrow C$ be the degree-2 branched cover defined by

$$
\begin{aligned}
& \left(3 t^{3}-t^{2}+t+1\right)\left(368 t^{4}-597 t^{3}-233 t^{2}+233 t+41\right) x^{2}+x\left(4\left(199 t^{4}-31 t^{3}-185 t^{2}-33 t+6\right) u\right. \\
& \left.\quad+2\left(430 t^{7}-1601 t^{6}+876 t^{5}-623 t^{4}-338 t^{3}+257 t^{2}-168 t-65\right)\right)
\end{aligned}
$$




$$
\begin{aligned}
& +4\left(138 t^{5}-153 t^{4}-21 t^{3}+55 t^{2}+3 t-18\right) u \\
& +552 t^{8}-1616 t^{7}-1435 t^{6}+4654 t^{5}-3949 t^{4}+900 t^{3}+1035 t^{2}-690 t+21=0 .
\end{aligned}
$$

The curve $Z$ is of genus 11 and admits a map $\psi$ to $C$ of degree 4 . The induced endomorphism $T=\psi_{*} \circ \phi^{*}$ of $\operatorname{Jac}(C)$ satisfies $T^{2}-2=0$ and generates real multiplication by $\mathcal{O}_{8}$.

REMARK 13. The curve $C$ in Theorem 12 corresponds to the point $(r, s)=\left(\frac{1}{8}, \frac{59}{32}\right)$ on $Y\left(\mathcal{O}_{8}\right)$ in the coordinates of [4]. Note that the coefficients of the polynomial $F$ defining $Z$ are not in $K(t)$, in contrast to the case where $C$ is a quintic hyperelliptic curve. This is because the hyperelliptic involution does not preserve the chosen point at infinity $P_{0}$, and therefore does not commute with the deck transformation of $\phi: Z \rightarrow C$. Therefore, the discovery part of our algorithm in which we compute equations for $Z$ has to be modified slightly. The coefficients of $F$ can be computed by determining a $K$-linear relation between $1, t\left(Q_{i}\right), t\left(Q_{i}\right)^{2}, u_{i}=u\left(P_{i}\right)$ and $u_{i} t\left(Q_{i}\right)$ by LLL for each $i$ (rather than between the first three quantities as in the quintic case). The coefficients in these relations are values of rational functions specialized at $t_{i}=t\left(P_{i}\right)$, and we can interpolate to determine these rational functions exactly. A similar modification must be made to solve for $y \in K(Z)$. For brevity, we have omitted the expression for $y$ here, although it is available in the computer files.

\section{Correspondences in families}

In this section we describe a correspondence on a universal family of genus- 2 curves over the entire Hilbert modular surface $Y\left(\mathcal{O}_{5}\right)$. There is one significant obstacle to implementing the method described in $\S 3$ in families. Suppose $\left\{C_{\mu}: \mu \in U\right\}$ is a family of curves parametrized by the base $U$ each of which admits real multiplication by $\mathcal{O}$. The method described in $\S 3$ allows us to compute a correspondence $Z_{\mu}$ over $C_{\mu}$ for any particular $\mu \in U$. However, the first step in computing $Z_{\mu}$ involves a choice of analytic Jacobian endomorphism $T_{\mu}^{\text {an }}$ generating $\mathcal{O}$. There are typically two choices for $T_{\mu}^{\text {an }}$ with a given minimal polynomial, and it is important to make these choices so that the matrices $T_{\mu, \Omega}^{\text {an }}$ vary continuously in $\mu$ and the $Z_{\mu}$ are fibers of a single family.

To overcome this obstacle we first normalize the entire family so that $d t / u$ and $t d t / u$ are eigenforms, using the eigenform location algorithm in [11]. Then we simply choose $T_{\mu}^{\text {an }}$ to have $T_{\mu, \Omega}^{\text {an }}$ equal to a constant diagonal matrix. Having consistently chosen $T_{\mu}^{\text {an }}$ in this way, we compute $Z_{\mu}$ for various values of $\mu$ and interpolate to determine a correspondence over the entire family. The result is the following theorem.

Theorem 14. For $(p, q) \in \mathbb{C}^{2}$, let $C(p, q)$ be the curve defined by the equation

$$
u^{2}=t^{6}+2 p t^{5}+10 q t^{3}+10 q^{2} t-5(p-1) q^{2}
$$

and let $\phi: Z(p, q) \rightarrow C(p, q)$ be the degree-2 branched cover defined by

$$
\begin{aligned}
& (2 t-p)(4 t+(3+\alpha) p) x^{2} \\
& \quad+\left(2(-\alpha-1) u+\alpha\left(2 t^{3}-2 p t^{2}+p^{2} t+2 q\right)-\left(6 t^{3}-6 p t^{2}-p^{2} t-10 q\right)\right) x \\
& \quad-2((1-\alpha) t-p) u+\alpha\left(2 t^{4}-p^{2} t^{2}+6 q t-4 p q\right) \\
& \quad-\left(2 t^{4}-2 p t^{3}+3 p^{2} t^{2}-10 q t+10 p q\right)=0,
\end{aligned}
$$

where $\alpha=\sqrt{5}$. For generic $(p, q) \in \mathbb{C}^{2}$, the curve $Z(p, q)$ is of genus 8 and admits a holomorphic map $\psi$ to $C(p, q)$ of degree 3. The endomorphism $T=\psi_{*} \circ \phi^{*}$ of $\operatorname{Jac}(C(p, q))$ is self-adjoint with respect to the Rosati involution and generates real multiplication by $\mathcal{O}_{5}$. 
The only complication in the proof of Theorem 14 is that we need to work in the function field over the base field $\mathbb{Q}(p, q)$ rather than $\mathbb{Q}$. We provide computer code in the auxiliary files to carry out the certification as in our previous examples. For brevity, we have omitted the lengthy expression for $y$ in the map $\psi:(t, u, x) \mapsto(x, y)$; it is available from the computer files. For that choice of $y$, the endomorphism $T$ has minimal polynomial $T^{2}-T-1$. (Replacing $y$ with $-y$ gives rise to an endomorphism with minimal polynomial $T^{2}+T-1$.)

REMARK 15. The coordinates for $Y\left(\mathcal{O}_{5}\right)$ in Theorem 14 are related to the coordinates $(m, n)$ appearing in [4] by

$$
(p, q)=\left(m^{2} / 5-n^{2},(m-\alpha n)\left(5 n^{2}-m^{2}\right)\left(5 n^{2}-m^{2}+5\right) / 125\right) .
$$

In particular, our coordinates are quadratic twists of those appearing in [4]. This is because they are adapted to the eigenform moduli problem, not the real multiplication moduli problem. The field of definition of a point $(p, q)$ is the field of definition of the eigenforms $d t / u$ and $t d t / u$, which need not agree with the field of definition of real multiplication. In fact, these moduli problems are isomorphic over $\mathbb{Q}(\sqrt{5})$, but not over $\mathbb{Q}$. This also explains the appearance of $\alpha=\sqrt{5}$ in the equation defining $Z(p, q)$.

\section{Divisor classes supported at eigenform zeros}

We now turn to the applications in dynamics for our equations for real multiplication stated in the introduction. Recall that $L$ is the multisection in the universal Jacobian over $\mathcal{M}_{2,1}\left(\mathcal{O}_{5}\right)$ whose values at the pointed curve $(C, P)$ are divisors of the form in equation (1.2). Our goal is to prove that the locus $\mathcal{M}_{2,1}\left(\mathcal{O}_{5} ; L\right)$ defined by the vanishing of $L$ is an irreducible surface in $\mathcal{M}_{2,1}$ and that $\mathcal{M}_{2,1}\left(\mathcal{O}_{5} ; L\right)$ is the closure of a complex geodesic for the Teichmüller metric.

Marking eigenform zeros. We start by passing to a cover of $\mathcal{M}_{2,1}\left(\mathcal{O}_{5}\right)$ on which we can describe the multisection $L$ in terms of sections. To that end we define $\mathcal{M}_{2}^{\text {ze }}\left(\mathcal{O}_{5}\right)$ to be the space of pairs $(C, Z)$ where $C \in \mathcal{M}_{2}\left(\mathcal{O}_{5}\right)$ and $Z \in C$ is a zero of an eigenform for real multiplication by $\mathcal{O}_{5}$. Similarly, we define $\mathcal{M}_{2,1}^{\text {ze }}\left(\mathcal{O}_{5}\right)$ to be the pointed version consisting of triples $(C, P, Z)$ with $(C, P) \in \mathcal{M}_{2,1}\left(\mathcal{O}_{5}\right)$ and $(C, Z) \in \mathcal{M}_{2}^{\text {ze }}\left(\mathcal{O}_{5}\right)$. Here we are allowing $Z=P$.

The space $\mathcal{M}_{2}^{\text {ze }}\left(\mathcal{O}_{5}\right)$ is birational to the Hilbert modular surface $Y\left(\mathcal{O}_{5}\right)$. To see this, fix $\gamma \in \mathcal{O}_{5}$ satisfying $\gamma^{2}-\gamma-1=0$. A point $(C, Z) \in \mathcal{M}_{2}^{\text {ze }}\left(\mathcal{O}_{5}\right)$ determines a Rosati invariant endomorphism $T_{\gamma}(C, Z)$ of $\operatorname{Jac}(C)$ by the requirement that the line of 1-forms on $C$ vanishing at $Z$ are $\gamma$-eigenforms for $T_{\gamma}(C, Z)$. The map $(C, Z) \mapsto\left(\operatorname{Jac}(C), T_{\gamma}(C, Z)\right)$ is birational. In particular, $\mathcal{M}_{2}^{\text {ze }}\left(\mathcal{O}_{5}\right)$ is an irreducible surface.

Sections. Let $\eta$ be the hyperelliptic involution on $C$. We can now define a section $L_{\gamma}$ of the universal Jacobian over $\mathcal{M}_{2,1}^{\text {ze }}\left(\mathcal{O}_{5}\right)$ by the formula

$$
L_{\gamma}(C, P, Z)=(P-Z)-T_{\gamma}(C, Z) \cdot(\eta(Z)-Z) \in \operatorname{Jac}(C) .
$$

Let $\mathcal{M}_{2,1}^{\text {ze }}\left(\mathcal{O}_{5} ; L_{\gamma}\right)$ denote the locus in $\mathcal{M}_{2,1}^{\text {ze }}\left(\mathcal{O}_{5}\right)$ where $L_{\gamma}$ vanishes. Similarly, we define $T_{1-\gamma}(C, Z), L_{1-\gamma}$ and $\mathcal{M}_{2,1}^{\text {ze }}\left(\mathcal{O}_{5} ; L_{1-\gamma}\right)$ by replacing $\gamma$ with its Galois conjugate $1-\gamma$. From the definition of the multisection $L$, it is clear the map forgetting $Z$ sends the union of $\mathcal{M}_{2,1}^{\text {ze }}\left(\mathcal{O}_{5} ; L_{\gamma}\right)$ and $\mathcal{M}_{2,1}^{\text {ze }}\left(\mathcal{O}_{5} ; L_{1-\gamma}\right)$ onto $\mathcal{M}_{2,1}\left(\mathcal{O}_{5} ; L\right)$. In fact, each of these spaces individually maps onto $\mathcal{M}_{2,1}\left(\mathcal{O}_{5} ; L\right)$ since the sections $L_{\gamma}$ and $L_{1-\gamma}$ are related by $L_{\gamma}(C, P, Z)=L_{1-\gamma}(C, P, \eta(Z))$. We record this fact in the following proposition.

Proposition 16. The space $\mathcal{M}_{2,1}^{\text {ze }}\left(\mathcal{O}_{5} ; L_{\gamma}\right)$ maps onto $\mathcal{M}_{2,1}\left(\mathcal{O}_{5} ; L\right)$. 
We will now use our equations for real multiplication to show that $\mathcal{M}_{2,1}^{\mathrm{ze}}\left(\mathcal{O}_{5} ; L_{\gamma}\right)$ is a section of $\mathcal{M}_{2,1}^{\mathrm{ze}}\left(\mathcal{O}_{5}\right) \rightarrow \mathcal{M}_{2}^{\mathrm{ze}}\left(\mathcal{O}_{5}\right)$.

Proposition 17. For each $(C, Z) \in \mathcal{M}_{2}^{\text {ze }}\left(\mathcal{O}_{5}\right)$, there is a unique solution $P \in C$ to the equation $L_{\gamma}(C, P, Z)=0$.

Proof. The uniqueness is easy and does not require our equations for real multiplication. If $P_{1}, P_{2}$ are solutions to $L_{\gamma}(C, P, Z)=0$, then $P_{1}-P_{2}$ is a principal divisor. Since the smooth genus-2 curve $C$ admits no degree- 1 rational map, we must have $P_{1}=P_{2}$.

The locus in $\mathcal{M}_{2}^{\text {ze }}\left(\mathcal{O}_{5}\right)$ consisting of pairs $(C, Z)$ which admit a solution to $L_{\gamma}(C, P, Z)=0$ is closed. This follows from the fact that $\mathcal{M}_{2,1}^{\text {ze }}\left(\mathcal{O}_{5}\right) \rightarrow \mathcal{M}_{2}^{\text {ze }}\left(\mathcal{O}_{5}\right)$ is a projective map and, since $\mathcal{M}_{2}^{\text {ze }}\left(\mathcal{O}_{5}\right)$ is irreducible, it is enough to check that the generic pair $(C, Z) \in \mathcal{M}_{2}^{\text {ze }}\left(\mathcal{O}_{5}\right)$ admits such a solution.

Recall the notation of Theorem 14 and its proof in the auxiliary files. For generic $(p, q) \in \mathbb{C}^{2}$, we have a genus- 2 curve $C(p, q)$, a Rosati invariant endomorphism $T(p, q)$ of $\operatorname{Jac}(C(p, q))$ satisfying $T(p, q)^{2}-T(p, q)-1=0$, and a $T(p, q)$-eigenform $\omega(p, q)=t d t / u$ with eigenvalue $\gamma=(1+\alpha) / 2$. To mark a zero of $\omega(p, q)$, we choose $z$ a square root of $5(1-p)$ and set

$$
Z(z, q)=(0, z q) \in C(p, q) .
$$

Counting dimensions, we see that the $(z, q)$-plane parametrizes an open subset of $\mathcal{M}_{2}^{\text {ze }}\left(\mathcal{O}_{5}\right)$ by the formula $(z, q) \mapsto(C(z, q), Z(z, q))$. We further define

$$
P(z, q)=\left(2(1-p), z\left(8-16 p+8 p^{2}+5 q\right) / \alpha\right) \in C(z, q) .
$$

Using our equations for the correspondence defining $T(z, q)$ and equation (2.3), we compute the divisor $T(z, q) \cdot(\eta(Z(z, q))-Z(z, q))$. Combined with standard formulas for the group law on $\operatorname{Jac}(C)$ (which have been implemented in Magma), we verify that $L_{\gamma}(C(z, q), P(z, q), Z(z, q)$ ) $=0$. We include code in the auxiliary files to verify this equation.

We are now ready to prove Theorem 2 .

Proof of Theorem 2. The locus $\mathcal{M}_{2,1}^{\text {ze }}\left(\mathcal{O}_{5} ; L_{\gamma}\right)$ is biregular to the irreducible surface $\mathcal{M}_{2}^{\text {ze }}\left(\mathcal{O}_{5}\right)$ by Proposition 17, and maps onto $\mathcal{M}_{2,1}\left(\mathcal{O}_{5} ; L\right)$ by a map of finite degree. Therefore $\mathcal{M}_{2,1}\left(\mathcal{O}_{5} ; L\right)$ is an irreducible surface in $\mathcal{M}_{2,1}$.

Complex geodesics in moduli space. We now prove Theorem 3 about geodesics in $\mathcal{M}_{2,1}$ which is a corollary of Theorem 2. We refer the reader to the survey articles $[\mathbf{2 3}, \mathbf{2 4}]$ for background on geodesics in the moduli space of curves.

Proof of Theorem 3. Fix a curve $C \in \mathcal{M}_{2}\left(\mathcal{O}_{5}\right)$ and an $\mathcal{O}_{5}$-eigenform $\omega$ on $C$. The form $\omega$ generates a complex geodesic $f_{\omega}: \mathbb{H} \rightarrow \mathcal{M}_{2}$ with $f_{\omega}(i)=C$ and $f_{\omega}^{\prime}(i)$ tangent to $\mathcal{M}_{2}\left(\mathcal{O}_{5}\right)$. By [12], the image of $f_{\omega}$ is contained in $\mathcal{M}_{2}\left(\mathcal{O}_{5}\right)$. We choose $C$ and $\omega$ generically so that $\overline{f_{\omega}(\mathbb{H})}=\mathcal{M}_{2}\left(\mathcal{O}_{5}\right)($ cf. $[\mathbf{1 3}])$.

The values of $f_{\omega}$ are related to $C$ by Teichmüller mappings. In particular, there is a distinguished holomorphic 1-form $\omega_{\tau}$ (up to scale) on $C_{\tau}=f_{\omega}(\tau)$ and a homeomorphism $C \rightarrow C_{\tau}$ which is affine for the singular flat metrics $|\omega|$ and $\left|\omega_{\tau}\right|$. The zeros of $\omega$ are in bijection with those of $\omega_{\tau}$ via the Teichmüller mapping and, by [12], $\omega_{\tau}$ is also an $\mathcal{O}_{5}$-eigenform. We conclude that there is a holomorphic zero marked lift

$$
f_{\omega}^{\mathrm{ze}}: \mathbb{H} \rightarrow \mathcal{M}_{2}^{\mathrm{ze}}\left(\mathcal{O}_{5}\right)
$$




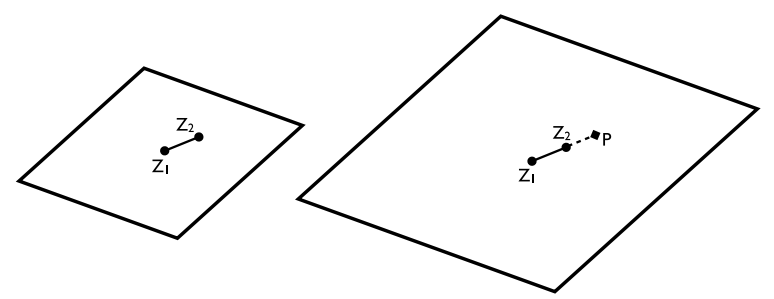

Figure 2. Genus-2 eigenforms for $\mathcal{O}_{5}$ can be built out of a parallelogram $U \subset \mathbb{C}$ and the similar parallelogram $\gamma U$ by a connected sum. The resulting form has zeros at $Z_{1}=0$ and $Z_{2}=t$ and the marked point $P=\gamma t$ satisfies $L_{\gamma}\left(C, P, Z_{1}\right)=0$.

whose composition with the map forgetting $Z$ equals $f_{\omega}$. Composing $f_{\omega}^{\text {ze }}$ with the section $\mathcal{M}_{2}^{\mathrm{ze}}\left(\mathcal{O}_{5}\right) \rightarrow \mathcal{M}_{2,1}^{\mathrm{ze}}\left(\mathcal{O}_{5} ; L_{\gamma}\right)$ and the map forgetting $Z$, we obtain a map

$$
f_{\omega}^{P}: \mathbb{H} \rightarrow \mathcal{M}_{2,1}
$$

which is a section of $f_{\omega}$ over $\mathcal{M}_{2,1} \rightarrow \mathcal{M}_{2}$.

There are several ways to conclude that $f_{\omega}^{P}$ is a complex geodesic. The map $f_{\omega}^{P}$ is a section over the complex geodesic $f_{\omega}$, and such sections are complex geodesics by a wellknown argument relying on the equality of the Kobayashi and Teichmüller metrics on $\mathcal{M}_{g, n}$. Alternatively, for $\left(C_{\tau}, P_{\tau}\right)=f_{\omega}^{P}(\tau)$ we have an $\mathcal{O}_{5}$-eigenform $\omega_{\tau}$ and a zero $Z_{\tau}$ of $\omega_{\tau}$ satisfying $L_{\gamma}\left(C_{\tau}, P_{\tau}, Z_{\tau}\right)=0$. We conclude that the relative periods

$$
\int_{Z_{\tau}}^{P_{\tau}} \omega_{\tau} \text { and } \gamma \int_{Z_{\tau}}^{\eta\left(Z_{\tau}\right)} \omega_{\tau}
$$

differ by an absolute period of $\omega_{\tau}$. Consequently, the Teichmüller mapping from $C \rightarrow C_{\tau}$ sends $P$ to $P_{\tau}$ and $f_{\omega}^{P}$ is a complex geodesic.

Thus we have a complex geodesic $f_{\omega}^{P}$ in $\mathcal{M}_{2,1}$ such that $\overline{f_{\omega}^{P}(\mathbb{H})}$ lies in $\mathcal{M}_{2,1}\left(\mathcal{O}_{5} ; L\right)$ and maps onto $\mathcal{M}_{2}\left(\mathcal{O}_{5}\right)$. Since both $\mathcal{M}_{2,1}\left(\mathcal{O}_{5} ; L\right)$ and $\mathcal{M}_{2}\left(\mathcal{O}_{5}\right)$ are irreducible surfaces, we must have $\overline{f_{\omega}^{P(\mathbb{H})}}=\mathcal{M}_{2,1}\left(\mathcal{O}_{5} ; L\right)$.

Polygons and marked points. McMullen described how to polygonally present eigenforms for $\mathcal{O}$ in genus $2[\mathbf{1 3}]$. Set $\gamma=(1+\sqrt{5}) / 2$ to be the golden mean. Eigenforms for discriminant 5 are obtained from a parallelogram $U \subset \mathbb{C}$ centered at 0 and the similar parallelogram $\gamma U \subset \mathbb{C}$ by gluing opposing sides on each parallelogram and performing a connected sum along a straight line interval $I$ connecting 0 and $t \in U$. The form $d z$ is invariant under these gluing maps and the resulting quotient $(C, \omega)=\left(U \#_{I} \gamma U, d z\right) / \sim$ is an $\mathcal{O}_{5}$-eigenform. Wright's conjecture of the existence of a dynamically natural way to mark curves in $\mathcal{M}_{2}\left(\mathcal{O}_{5}\right)$ posited in particular that one could mark the eigenform $\left(U \#_{I} \gamma U\right) / \sim$ at the point $P=\gamma t$ in the polygon $\gamma U$ (see Figure 2).

One way to see that the algebraically presented locus $\mathcal{M}_{2,1}\left(\mathcal{O}_{5} ; L\right)$ in Theorem 3 equals the locus polygonally presented by Wright is by first checking that they agree somewhere, for example at the regular decagon eigenform which is the limit of $(C(z, q), P(z, q))$ as $q \rightarrow 0$ in our parametrization. The period relations imposed by the vanishing of $L$ then imply that the points marked in $\mathcal{M}_{2,1}\left(\mathcal{O}_{5} ; L\right)$ coincide with Wright's polygonal description at a nearby generic point. Therefore, the algebraic and polygonal descriptions agree along an entire complex geodesic which is dense in $\mathcal{M}_{2,1}\left(\mathcal{O}_{5} ; L\right)$. 
Acknowledgements. We thank Curt McMullen for many helpful conversations and suggestions, Noam Elkies and Alex Wright for useful comments on an earlier draft of this paper, and the anonymous referees for a careful reading of the paper and numerous helpful comments. REM was supported in part by National Science Foundation grant DMS-1103654.

\section{References}

1. C. Birkenhake and H. Lange, Complex abelian varieties, 2nd edn, Grundlehren der mathematischen Wissenschaften 302 (Springer, Berlin, 2004).

2. W. Bosma, J. J. Cannon, C. Fieker and A. Steel (eds), Handbook of Magma functions, available online at http://magma.maths.usyd.edu.au/magma/handbook/.

3. J. W. S. CASSEls and E. V. Flynn, Prolegomena to a middlebrow arithmetic of curves of genus 2, London Mathematical Society Lecture Note Series 320 (Cambridge University Press, Cambridge, 1996).

4. N. D. Elkies and A. Kumar, 'K3 surfaces and equations for Hilbert modular surfaces', Algebra Number Theory 8 (2014) no. 10, 2297-2411.

5. A. Eskin, C. T. McMullen, R. E. Mukamel and A. Wright, 'Billiards in quadrilaterals, Hurwitz spaces, and real multiplication of Hecke type', in preparation.

6. S. Filip, 'Splitting mixed Hodge structures over affine invariant manifolds', Ann. of Math. (2) 183 (2016) $681-713$.

7. K. Налнімото and Y. SAKAI, 'On a versal family of curves of genus two with $\sqrt{2}$-multiplication', Algebraic number theory and related topics 2007, RIMS Kôkyûroku Bessatsu B12 (Res. Inst. Math. Sci. (RIMS), Kyoto, 2009) 249-261.

8. K. Hashimoto and Y. SAKai, 'General form of Humbert's modular equation for curves with real multiplication of $\Delta=5$ ', Proc. Japan Acad. Ser. A Math. Sci. 85 (2009) no. 10, 171-176.

9. G. Humbert, 'Sur les fonctionnes abéliennes singulières', J. Math. Pures Appl. série 5 t. V (1899) 233-350.

10. В. ЈАКов, 'Poncelet 5-gons and abelian surfaces', Manuscripta Math. 83 (1994) no. 2, 183-198.

11. A. Kumar and R. E. Mukamel, 2014 Algebraic models and arithmetic geometry of Teichmüller curves in genus two', Preprint, 2014, arXiv:1406.7057.

12. C. T. McMullen, 'Billiards and Teichmüller curves on Hilbert modular surfaces', J. Amer. Math. Soc. 16 (2003) 857-885.

13. C. T. McMullen, 'Dynamics of $\mathrm{SL}_{2}(\mathbb{R})$ over moduli space in genus two', Ann. of Math. (2) 165 (2007) $397-456$.

14. Y. SAKAI, 'Poncelet's theorem and curves of genus two with real multiplication of $\Delta=5$ ', J. Ramanujan Math. Soc. 24 (2009) no. 2, 143-170.

15. Y. SAKAI, 'Construction of genus two curves with real multiplication by Poncelets theorem', Dissertation, Waseda University, 2010.

16. B. A. Sмiтh, 'Explicit endomorphisms and correspondences', PhD Thesis, University of Sydney, 2005.

17. G. VAN DER Geer, Hilbert modular surfaces, Ergebnisse der Mathematik und ihrer Grenzgebiete (3) 16 (Springer, Berlin, 1988).

18. P. B. VAN WAMElen, 'Proving that a genus 2 curve has complex multiplication', Math. Comp. 68 (1999) no. 228, 1663-1677.

19. P. B. van WAMELEN, 'Computing with the analytic Jacobian of a genus 2 curve', Discovering mathematics with Magma (Springer, Berlin, 2006) 117-135.

20. P. B. VAN WAMELEN, 'Poonen's question concerning isogenies between Smart's genus 2 curves', Math. Comp. 69 (2000) no. 232, 1685-1697.

21. J. Wilson, 'Curves of genus 2 with real multiplication by a square root of 5', DPhil Thesis, Oxford University, 1998.

22. J. Wilson, 'Explicit moduli for curves of genus 2 with real multiplication by $\mathbf{Q}(\sqrt{5})$ ', Acta Arith. 93 (2000) no. 2, 121-138.

23. A. Wright, 'Translation surfaces and their orbit closures: An introduction for a broad audience', EMS Surv. Math. Sci. 2 (2015) 63-108.

24. A. Zorich, 'Flat surfaces', On random matrices, zeta functions and dynamical systems, Frontiers in Number Theory, Physics and Geometry 1 (Springer, Berlin, 2006) 439-586.

\section{Abhinav Kumar \\ Department of Mathematics \\ Stony Brook University \\ Stony Brook, NY 11794 \\ USA}

thenav@gmail.com

\author{
Ronen E. Mukamel \\ Department of Mathematics \\ Rice University MS 136 \\ 6100 Main St. \\ Houston, TX 77005 \\ USA
}

ronen@rice.edu 